EPJ Web of Conferences 59, 08002 (2013)

DOI: $10.1051 /$ epjconf/20135908002

(C) Owned by the authors, published by EDP Sciences, 2013

\title{
Active mirror amplifiers for HiPER kiloJoule beamlines
}

\author{
J.-C. Chanteloup and A. Lucianetti
}

LULI, Ecole Polytechnique, CNRS, CEA, UPMC, 91128 Palaiseau, France

\begin{abstract}
A major challenge the HiPER [1] project is facing is to derive laser architectures satisfying simultaneously all HiPER requirements; among them, high wall-plug efficiency (15 to 20\%) and repetition rate (around $10 \mathrm{~Hz}$ ) are the most challenging constraints. Several groups over the world are actively pursuing research in the field of High average power Diode Pumped Solid State Lasers (DPSSL) [2]. We propose a comprehensive solution for a $1 \mathrm{~kJ}$ DPSSL beamline as the unit brick of a 12 beams bundle.
\end{abstract}

\section{INTRODUCTION}

HiPER (High Power laser Energy Research) [1, 3, 4] in Europe, LIFE (Laser Inertial Fusion Engine) [5] in the United States of America and GENBU (Generation of ENergetic Beam Ultimate) [6] in Japan are scientific programs dedicated to demonstrate the feasibility of laser driven fusion [1] as a future energy source.

The Centre National de la Recherche Scientifique (CNRS) Laboratoire pour l'Utilisation des Lasers Intenses (LULI) at Ecole Polytechnique, Palaiseau, France is working on a HiPER scheme relying on $\mathrm{Yb}^{3+}$ :YAG cryo-cooled active mirror amplifiers. Six amplifiers in a double pass configuration will be required to reach the $1 \mathrm{~kJ}$ unit beam (called "Beamlet") requirement for HiPER. Considering the state of the art coating maximum Laser Induced Damage Threshold ( $\sim 30$ to $40 \mathrm{~J} / \mathrm{cm}^{2}$ for $1030 \mathrm{~nm}$, ns regime), the component lifetime requirements $\left(10^{9}\right.$ shots $)$ and a reasonable damage safety factor, it seems safe to target maximum extraction fluence around $10 \mathrm{~J} / \mathrm{cm}^{2}$. Therefore, about $\sim 1 \mathrm{~kJ}$ per beam leads to an aperture of about $100 \mathrm{~cm}^{2}$.

A single beamline (see arrangement of 12 beamlets on figure 1) will be based on 9 beamlets to be incoherently combined for compression pulses. And, considering the requirements in terms of spot size, the 3 remaining beamlets will be coherently combined for shock ignition pulses. The laser requirements are summarized in the table of figure 1.

\section{OPTICAL LAYOUT}

Figure 2 displays a schematic of the proposed 2-pass amplifying chain for HiPER. The laser beam passes through the amplifier system twice after reflection on a deformable mirror (left): it travels through each disk four times. Six of these amplifiers will be used in series in order to reach the requested energy level.

Figure 4 shows an alternative triangular configuration where the footprint would be approximately $12 \times 12 \mathrm{~m}^{2}$.

This is an Open Access article distributed under the terms of the Creative Commons Attribution License 2.0, which permits unrestricted use, distribution, and reproduction in any medium, provided the original work is properly cited. 
EPJ Web of Conferences

\begin{tabular}{|c|c|c|c|c|c|}
\hline & $\begin{array}{c}\text { Total energy } \\
(\mathrm{kJ})\end{array}$ & Spots & $\begin{array}{c}\text { Energy per } \\
\text { spot }(\mathrm{kJ})\end{array}$ & $\begin{array}{c}\text { Beam per } \\
\text { bundle }\end{array}$ & $\begin{array}{c}\text { Energy per } \\
\text { beam }(\mathrm{kJ})\end{array}$ \\
\hline Compression & 500 & 48 & 10,42 & 9 & 1,16 \\
\hline Shock Ignition & 130 & 48 & 2,71 & 3 & 0,90 \\
\hline Total & 630 & 48 & 13,125 & 12 & - \\
\hline
\end{tabular}


Figure 1. HiPER shock ignition scheme relies on 48 spots on the target. The $630 \mathrm{~kJ}$ total energy will therefore have to be delivered through 48 bundles of twelve 0.9 to $1.16 \mathrm{~kJ}$ beams.

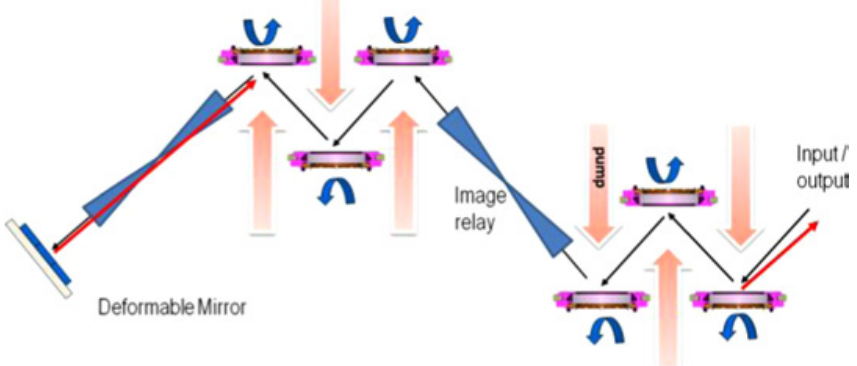

Figure 2. Schematic of a six disk Yb:YAG amplifying chain, where the laser beam passes through the amplifier system twice and through each disk four times.

kJ HiPER beamline (LULI/CNRS)



Figure 3. Triangular configuration for $1 \mathrm{~kJ}$ HiPER beamlet. 
Table 1. Parameters used in the parametric study.

\begin{tabular}{|l|l|l|}
\hline$\lambda_{\mathrm{p}}$ & $940 \mathrm{~nm}$ & Pump wavelength \\
\hline$\lambda_{\mathrm{s}}$ & $1030 \mathrm{~nm}$ & Laser wavelength \\
\hline$\sigma_{\text {ems_L }}$ & $5.710^{-20} \mathrm{~cm}^{2}$ & Laser emission cross section \\
\hline$\sigma_{\text {abs_L }}$ & $5.810^{-22} \mathrm{~cm}^{2}$ & Laser absorption cross section \\
\hline$\sigma_{\text {ems_p }}$ & $9.7510^{-22} \mathrm{~cm}^{2}$ & Pump emission cross section \\
\hline$\sigma_{\text {abs_p }}$ & $1.310^{-20} \mathrm{~cm}^{2}$ & Pump absorption cross section \\
\hline$\tau_{\mathrm{f}}$ & $1 \mathrm{~ms}$ & Fluorescence time \\
\hline $\mathrm{I}$ & 4 to $8 \mathrm{~kW} / \mathrm{cm}^{2}$ & Pump intensity \\
\hline$\tau_{\mathrm{p}}$ & $0.7 \mathrm{~ms}$ & Pump duration \\
\hline $\mathrm{L}$ & 1 to $2.5 \mathrm{~cm}$ & Crystal thickness \\
\hline $\mathrm{D}$ & $11 \mathrm{~cm}$ & Rectangular aperture smaller size \\
\hline$\theta$ & 0 to $40^{\circ}$ & Extraction angle \\
\hline $\mathrm{d}$ & 0.1 to 0.2 at $\%$ & Doping level \\
\hline
\end{tabular}

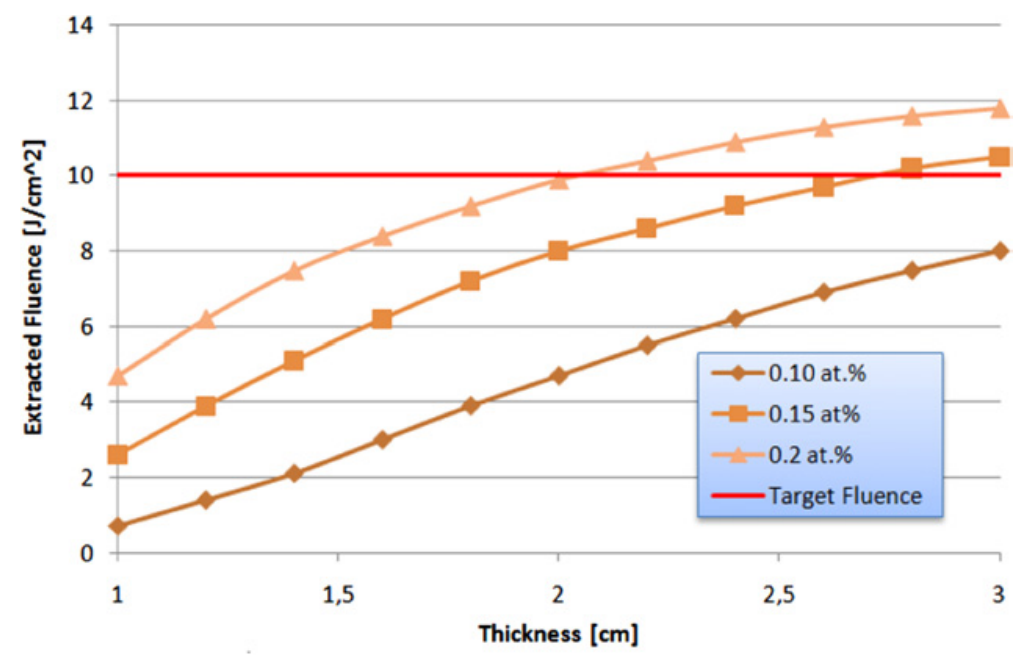

Figure 4. Extracted fluence (in $\left.\mathrm{J} / \mathrm{cm}^{2}\right)$ versus the thickness for three doping concentrations $(0.1 \mathrm{at} \%, 0.15 \mathrm{at} \%$, and $0.2 \mathrm{at} \%)$ and the thickness of the thin disk for $\mathrm{I}_{\mathrm{P}}=6 \mathrm{~kW} / \mathrm{cm}^{2}$.

\section{EXTRACTED FLUENCE}

We have explored the influence of different pump and disk parameters. Having fixed the aperture $\left(\sim 100 \mathrm{~cm}^{2}\right)$ and the extraction fluence $\left(\sim 10 \mathrm{~J} / \mathrm{cm}^{2}\right)$, we have performed a parametric study with parameters and limits given in table 1 and resulting optimum point of operation in section 4 .

Thermal management [7] and Amplified Spontaneous Emission management [8] solutions proposed within the LULI Lucia program framework helped us deriving an optimum point of operation. Figure 4 displays the resulting extracted fluence for $\mathrm{I}_{\mathrm{P}}=6 \mathrm{~kW} / \mathrm{cm}^{2}$.

\section{CONCLUSION}

For doping concentration of 0.15 at $\%$, thickness about $2.6 \mathrm{~cm}$, and incident angle of $22^{\circ}$ we are able to mitigate ASE parasitic oscillations. The corresponding extracted energy is close to $10 \mathrm{~J} / \mathrm{cm}^{2}$ as 
illustrated on figure 4 and the optical efficiency is well above $35 \%$. The resulting optimum point of operation is derived for 2 passes through 2 amplifiers of 3 disks each:

- Extraction angle : $22^{\circ}$

- Aperture : $11 \times 11.7=129 \mathrm{~cm}^{2}$

- Final extraction Fluence $: 10 \mathrm{~J} / \mathrm{cm}^{2}$

- Energy extracted : $1.3 \mathrm{~kJ}$

- Pump intensity : $6 \mathrm{~kW} / \mathrm{cm}^{2}$

- Yb Doping level : 0.15 at\%

Luli is currently building a 10 to 30 Joules scale prototype aiming at addressing several of key laser physics issues (like thermal and ASE management) related to the laser driver HiPER proposal presented here.

The authors also gratefully acknowledge the support of the MŠMT, Ministry of Education, Youth and Sports of the Czech Republic in supporting this work through the HiPER program.

\section{References}

[1] M. Dunne, Nature Physics, 2(1), 2-5 (2006)

[2] J.-C. Chanteloup and D. Albach, IEEE Photonics Journal, 3(2) (2011)

[3] J.-C. Chanteloup, D. Albach, A. Lucianetti, K. Ertel, S. Banerjee, P. Mason, C.Hernandez-Gomez, J. Collier, J. Hein, M. Wolf, J.Körner and B. Le Garrec, 2010 Journal of Physics: Conference Series, 244(1), 012010 (2010)

[4] B. Le Garrec, C. Hernandez-Gomez, T. Winstone and J. Collier, 2010 Journal of Physics: Conference Series, 244(3), 032020 (2010)

[5] A. Bayramian, R. Campbell, C. Ebbers, B. Freitas, J. Latkowski, W. Molander, S. Sutton, S. Telford and J. Caird, 2010 Journal of Physics: Conference Series, 244(3), 032016 (2010)

[6] H. Furuse, Y. Takeuchi, T. Nakanishi, A. Yoshida, R. Yasuhara, T. Kawashima, H. Kan, N. Miyanaga and J. Kawanaka, 6th International workshop on High Energy Class Diode Pumped Solid State Laser, Versailles, France, 8-10 Sept. 2010 (2010).

[7] D. Albach, G. Le Touzé, and J.-C. Chanteloup, Optics Express, 19(9), 8413-8422 (2011)

[8] D. Albach, J.-C. Chanteloup and G. Le Touzé, Optics Express, 17(5), 3792-3801 (2009) 